\title{
Leucocytoclastic vasculitis in a patient with azathioprine hypersensitivity
}

\author{
CG Beckett, P Hill, KR Hine
}

\begin{abstract}
Summary
We report the case of a 77-year-old man admitted nine days after being commenced on azathioprine with symptoms initially thought to be secondary to sepsis but in fact due to azathioprine hypersensitivity. He developed histologically proven cutaneous leucocytoclastic vasculitis following the re-introduction of azathioprine. We review the literature concerning adverse reactions to azathioprine and the problems of making the diagnosis as well as highlighting azathioprine as a novel cause of leucocytoclastic vasculitis.
\end{abstract}

Keywords: azathioprine, leucocytoclastic vasculitis, drug hypersensitivity

A 77-year-old man presented with a 48-hour history of fever and malaise nine days after being commenced on azathioprine. Nine months previously he presented to his general practitioner with easy bruising, and a routine blood count found him to have a platelet count of $6 \times 10^{9} / 1$. Following referral to the local haematologist, bone marrow examination was compatible with the diagnosis of immune thrombocytopenia. Eight years previously he was found to have a small transitional cell carcinoma of the bladder following an episode of haematuria. Regular follow-up cytoscopies have shown no evidence of recurrence. Blood tests performed at the time found a kappa IgG monoclonal gammopathy. Despite prednisolone $10 \mathrm{mg} /$ day, his platelet count ran between $60-80 \times 10^{9} / 1$ and so azathioprine $50 \mathrm{mg}$ tid was added nine days prior to admission.

On admission, other than a pyrexia and a grade 1/6 pan systolic murmur, nothing else was found. Chest X-ray was unremarkable. Blood and urine cultures were sent and cefotaxime $1 \mathrm{~g}$ tid commenced. Initial white cell count was $10.3 \times 10^{9} / 1$ and platelet count $102 \times 10^{9} / 1$. There was no evidence of neutropenia but because sepsis was suspected, azathioprine was withheld. The following morning he was apyrexial and so azathioprine was re-introduced. The next day he complained of a widespread but faint macular rash, low back pain and his pyrexia had recurred. It was thought that the rash was secondary to the cefotaxime, and so his antibiotics were changed to piperacillin and gentamicin.

Unfortunately, he remained unwell over the weekend and early on Monday morning he developed atrial fibrillation with fast ventricular response and resultant cardiovascular compromise requiring DC cardioversion to restore sinus rhythm. Serum and urine electrolytes revealed that he had developed acute renal failure, with serum creatinine peaking at $700 \mu \mathrm{mol} / 1$. On examination, the rash had become papular and tender and there were nail-fold lesions. All medications were stopped other than the prednisolone. The patient's observation chart demonstrated that he had a temperature of $39^{\circ} \mathrm{C}$ on admission but became apyrexial in the first $24 \mathrm{~h}$ when azathioprine was withheld, only for the pyrexia to recur on re-introduction of azathioprine. When the azathioprine was finally stopped, the patient remained apyrexial for the remainder of the admission. A dermatological opinion confirmed that this was a vasculitic rash. Skin biopsy was performed and histology demonstrated a leucocytoclastic vasculitis of small dermal vessels (figure).

Over the following two weeks renal function returned to normal and the rash resolved. On advice from a renal unit it was felt that renal biopsy should not be performed unless renal function did not return to normal. The possible causes for the renal failure included allergic interstitial nephritis, necrotising glomerulonephritis due to renal vasculitis and renal failure due to arrhythmia-induced hypotension.

Extensive investigation for an infective cause including blood and urine culture and serology for enterovirus, cytomegalovirus, EpsteinBarr, parvovirus, toxoplasma and mycoplasma were negative. Autoimmune, complement, cryoglobulin, ANCA and immune complex serology were unremarkable. Again a kappa IgG monoclonal gammopathy was detected

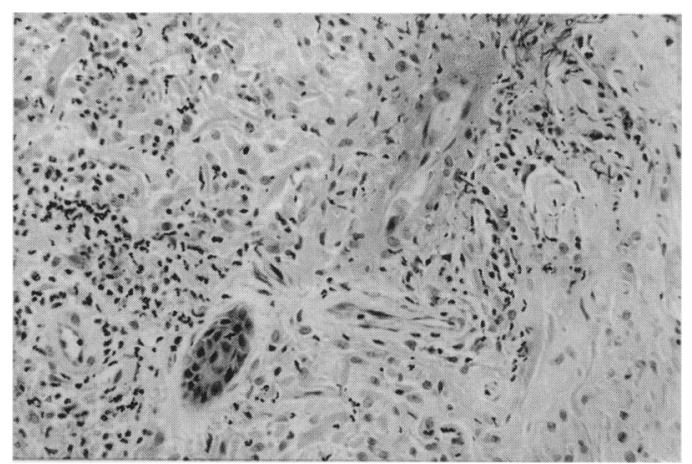

Figure Skin biopsy $(\times 200)$ demonstrating the leucocytoclastic vasculitis of small dermal vessels 
with urine Bence Jones protein not detected. Echocardiogram revealed minimal mitral regurgitation and no vegetations.

The man remains well six months after discharge with normal renal and cardiac function and his immune thrombocytopenia is controlled with prednisolone.

\section{Discussion}

Azathioprine has been used for 30 years in a variety of 'autoimmune' conditions such as systemic lupus erythematosus, rheumatoid arthritis and inflammatory bowel disease, as well as an immunosuppressant in organ transplantation. It is an oral purine analogue which is biologically inactive until metabolised to 6mercaptopurine and excretion is via the kidneys. The exact mechanism of action is not clear. The most common adverse effects are gastrointestinal disturbances in up to $60 \%$ of cases. ${ }^{1}$ Box 1 lists the possible manifestations of azathioprine hypersensitivity. The serious but much less common side-effect of reversible neutropenia has been reported in up to $7 \%$ of cases and thus warrants regular haematological monitoring in patients on treatment.

In all case reports, hypersensitivity symptoms commenced within three weeks of azathioprine being commenced. It is often the case that the diagnosis of azathioprine hypersensitivity is not suspected for some days as the symptoms are attributed to sepsis, especially in the context of Crohn's disease. ${ }^{2}$ In the case of organ transplantation, rejection has to be excluded. ${ }^{3}$ The other reports involve the use of azathioprine in patients with dermatological ${ }^{4}$ and rheumatological ${ }^{5}$ conditions which report transient disturbance of renal and hepatic function. There was only one previous letter found in the literature reporting a leucocytoclastic cutaneous vasculitis, ${ }^{6}$ in two patients with chronic renal failure, but there are few details regarding the exclusion of an infective cause. The Committee for Safety of Medicines cites two reports of a possible vasculitis associated with azathioprine but no further evidence was given. A literature review found no reports of vasculitis associated with immune thrombocytopenia, although a review of cases of urticarial vasculitis in a connective disease clinic found one person with an IgG paraproteinaemia.

This case demonstrates that azathioprine is a cause of cutaneous leucocytoclastic vasculitis. It emphasises the fact that sepsis can delay the diagnosis of azathioprine-related hypersensitivity reaction and that an infective agent needs to be excluded as a cause of leucocytoclastic vasculitis. As azathioprine is commonly used for the treatment of systemic lupus erthyematosus and the maintenance of remission of

1 Singh G, Fries JF, Spitz P, et al. Toxic effects of azathioprine in rheumatoid arthritis. Arthritis Rheum 1989; 32: 837-43.

2 Wilson BE. Azathioprine hypersensitivity mimicking sepsis in Crohn's disease. Clin Infect Dis 1993; 17: 940-1.

3 Saway PA, Heck LW, Bonner JR, et al. Azathioprine hypersensitivity. Am $\mathcal{F}$ Med 1988; 84: 960-4.

4 Dodd HJ, Tatnall FM, Sarkany I. Fast atrial fibrillation induced by treatment of psoriasis with azathioprine. $B M \mathcal{F}$ 1985; 291: 706 .
Possible manifestations of azathioprine hypersenstivity

- fever: isolated with rigors

- musculoskeletal: arthralgias, myalgias, muscle weakness

- cutaneous: exanthematous eruption, urticaria and angioedema, erythema (multiforme-like and nodosum-like)

- neurological: headache, neuropathy

- cardiovascular: hypotension, arrhythmias

- renal: oliguria, interstitial nephritis

- gastrointestinal: nausea and vomiting, diarrhoea, cholestatic jaundice, hepatocellular injury, pancreatitis

- respiratory: dyspnoea, cough, pneumonitis

Box 1

\section{Causes of leucocytoclastic vasculitis}

Coexistent disease

- rheumatoid arthritis

- malignancy

- polyarteritis nodosa

- Sjogren's syndrome

- systemic lupus erythematosus

- Wegener's granulomatosis

- cryoglobulinaemia

Precipitating agents

- drugs: penicillin, thiazide diuretics, gold, erythromycin, allopurinol, amiodarone

- bacteria: Streptococcus, Escherichia coli

- viruses

Uncertain

- idiopathic

- chronic urticaria

- Henoch-Schonlein purpura

Box 2

\section{Summary points}

- azathioprine is a cause of leucocytoclastic vasculitis

- drug hypersenstivity reactions occur within three weeks of the introduction of the drug

- there is often a delay in diagnosis of azathioprine hypersensitivity as sepsis has to be excluded

- potentially fatal effects of drug rechallenge in a patient with a suspected drug hypersensitivity reaction

Box 3

patients with a primary systemic vasculitis, the distinction between relapse and azathioprine $\stackrel{\infty}{\rightarrow}$ hypersensitivity would be especially difficult. Finally, this case highlights the dangers of rechallenging a patient with a suspected drug hypersensitivity reaction. Jeurissen MEC, Boerbooms AM Th, Van de Putte LBA, et $\frac{O}{0}$ al. Azathioprine induced fever, chills, rash and hepatotoxicity in rheumatoid arthritis. Ann Rheum Dis 1990; 49: 25-7.

6 Bergman SM, Krane NK, Leonard G, et al. Azathioprine and hypersensitivity vasculitis. Ann Intern Med 1988; 109: 83-4. 